\title{
Effects of attentive encoding on analytic and nonanalytic processing in implicit and explicit retrieval tasks
}

\author{
LINDA J. ANOOSHIAN \\ Trinity University, San Antonio, Texas
}

\begin{abstract}
The least-frequent meanings of homophones were biased by two exposure conditions that varied in the extent to which the processing of biasing information was attentive and effortful. Memory was assessed with both explicit (recognition) and implicit tasks. For the implicit task, scores reflected the extent to which the biased meanings were apparent in subjects' later spellings of or free associatons to old homophones. The results indicate that attentive processing during encoding is likely to invite analytic processing in both implicit and explicit tasks. Furthermore, unattended exposure may lead to nonanalytic processing across both tasks, with subjects relying on a fluency heuristic for the explicit task. Comparable results were obtained from the spelling and the free-association tasks.
\end{abstract}

Most memory research has relied on tasks with explicit demands for remembering. In traditional recognition and recall tasks, for example, subjects are explicitly asked to retrieve information about prior episodes. In contrast, implicit tasks typically involve demonstrating that subjects' performances are affected by previous experiences even though the subjects are unaware of their own remembering. Recent research suggests that different processes and/or structures are used when memory is assessed with an implicit rather than explicit task (see Schacter, 1987). Unlike explicit tasks, performances on implicit tasks appear largely unaffected by level of processing (Jacoby \& Dallas, 1981) or the extent of elaborative processing (see Schacter \& Graf, 1986).

Jacoby and his colleagues (Jacoby, 1988; Jacoby \& Brooks, 1984) have argued that performances on both types of tasks rely on episodic representations, but that implicit tasks usually demand global nonanalytic processing while explicit tasks usually demand analytic processing in retrieving the original encoding context. Yet, under some conditions, performances on the two types of tasks seem to rely on the same type of processing. For example, a recognition judgment can reflect a nonanalytic "feeling of familiarity" based on the increased fluency of processing that results from similar processing in a prior episode (Jacoby, 1988). A variety of experimental manipulations could presumably affect the relative extent to which subjects rely on analytic versus nonanalytic processes for either implicit or explicit tasks.

For the present experiment, the primary manipulation was that of varying the extent of effortful or attentive processing during encoding. The two exposure conditions

I thank Paula T. Hertel for her thoughtful comments on earlier versions of the manuscript. Requests for reprints may be directed to Linda J. Anooshian, Department of Psychology, Boise State University, Boise, ID 83725 . were comparable to those used in previous studies with implicit and explicit tasks following either unattentive (Eich, 1984) or attentive processing (Jacoby \& Witherspoon, 1982). The expectation was that the more attentive the processing during encoding, the more likely that analytic processing would be evoked during retrieval.

\section{METHOD}

\section{Subjects}

A total of 24 subjects ( 12 males and 12 females) were assigned to each of four groups differentiated by exposure condition and type of implicit task. All subjects were college students who received extra course credit for participation.

\section{Word Selections}

A list of $\mathbf{4 8}$ homophones was constructed for which least- and mostfrequent meanings could be clearly identified from published norms $(\mathrm{Gal}-$ braith \& Taschman, 1969) and pilot testing. The homophones were divided randomly into three equal sets: those presented in exposure (old items for later retrieval tasks), new items (distractors) for the explicit task (recognition), and new items for the implicit tasks. A total of 56 nonhomophones, comparable to the overall set of homophones in word length and type, were randomly designated as filler items for the exposure task (8), distractors for the recognition task (32), or fillers for the implicit tasks (16).

\section{Procedure}

Unattentive encoding was achieved with a dichotic-listening condition in which the subjects shadowed a story while phrases containing the least-frequent meanings of homophones were presented in the unattended channel (e.g., "a fairy tale"). The subjects were told to repeat the story aloud and verbatim, and that they would be tested for their exact recall of the story. Short phrases were presented at 6-sec onset intervals in the unattended channel. Each of 16 biasing phrases for homophones was presented eight times, with different random orders for each block of 16 phrases. These eight blocks of biasing phrases were preceded, as well as followed, by 8 comparable phrases containing nonhomophones.

The question condition required attentive processing during exposure; the subjects answered questions that directed them to the least-frequent meanings of homophones (e.g., "Would you rather hear a fairy tale or a sad story?'). The 16 biasing questions for homophones were intermixed randomly with 8 comparable questions without homophones. One option for answering each question always corresponded to a specific 
biasing phrase constructed for the dichotic-listening condition (e.g., "a fairy tale').

All materials for these exposure tasks and later recognition tasks were tape-recorded in the same male and female voices. For the dichoticlistening condition, the voice of presentation (male or female) was different for the attended and unattended channels. For the question condition, the biasing phrases (and comparable phrases for nonhomophones) were recorded in a different voice than was the rest of the question. These procedures highlighted the biasing information and insured that later recognition tests would be equally stringent for the two exposure groups. That is, during recognition tests, the subjects were told to identify words that had been spoken by a particular speaker (male or female); they were not held accountable for every word presented in either the stories (dichotic-listening condition) or questions (question condition). Voice assignment was systematically varied within each exposure condition.

Following initial exposure and a brief filler task, each subject was tested for recognition of half of the 16 homophones for which biasing information had been presented during exposure. For each recognition trial, the subjects were instructed to identify the word previously heard in a particular voice (male or female). The three options presented for each trial had been recorded in that same voice (also the same voice used in recording the biasing phrases during exposure). For the 8 trials that included an old homophone, the two distractors included another homophone choice. Additional trials included an old filler item (4 trials) or presented options of three new items with a homophone choice $(8$ trials). These different types of recognition trials allowed for later assessments of whether correct recognition was inflated by the subjects who, when in doubt, used a strategy of choosing homophones over nonhomophones. (Later analyses indicated that the subjects were no more likely to select new homophones than new nonhomophones.) Recognition trials and options within trials were randomly arranged.

Finally, each subject received one of two implicit tasks. For each task, the subject heard a mixed list of 16 old homophones (presented during exposure), 16 new homophones, and 16 new nonhomophones. The subject was asked to spell or to free associate to each word as quickly as possible after it was presented. The subjects' free associations were terminated as soon as the interpreted meaning was clearly apparent (e.g., the subject said "story" and "fiction" after hearing "tale").

\section{RESULTS AND DISCUSSION}

The primary dependent measures were the number of correct recognition choices and the number of spellings or free associations that reflected the least-frequent meanings of homophones (for both old and new homophones). These measures were analyzed with ANOVA designs that included between-subjects factors for exposure condition and type of implicit task. When a within-subject factor was included in the design (e.g., old vs. new homophones), the multivariate approach to within-subject designs was used. The significance level for all analyses was set at .05 .

Overall performances on the implicit and explicit tasks were consistent with the pattern of results reported by Eich (1984) and by Jacoby and Witherspoon (1982). That is, both explicit and implicit performances were better for the question than the dichotic-listening condition. The subjects in the dichotic-listening condition recognized 4.67 (of 8 ) old homophones, while the subjects in the question condition recognized $5.67[F(1,92)=11.92, M S e=$ 2.01]. Whether free-associating or spelling, the subjects gave responses that were more in line with the biased meanings of old homophones in the question condition ( $M=7.15$ out of 16$)$ than in the dichotic-listening condition $(M=6.12)$. An ANOVA that included a withinsubject factor for homophone (old vs. new) yielded a main effect for homophone $[F(1,92)=12.25, M S e=3.52]$; least-frequent meanings were more apparent for old $(M$ $=6.64)$ than new homophones $(M=5.68)$. As expected, a significant interaction between exposure condition and homophone $[F(1,92)=5.51]$ reflected that this difference was greater for the question than for the dichoticlistening condition. Performance on spelling and free association did not vary as a function of whether items had appeared in the previous recognition test.

Further analyses were designed to examine the extent of stochastic independence between performances on the explicit and implicit tasks. Specifically, the question was whether the ability to remember a particular item in one fashion (e.g., explicitly) was predictive of the probability of remembering that same item in a different fashion (e.g., implicitly). For the 8 homophones included in both tasks, the proportion of correct responses for the implicit task (i.e., spellings or free associations reflecting biased meanings) was calculated separately for items recognized and those not recognized. An ANOVA that included a within-subject factor for implicit proportion (correct vs. incorrect recognition) revealed a significant interaction between exposure group and implicit proportion $[F(1,92)$ $=5.04, \mathrm{MSe}=.124]$. Only for the question condition did the subjects show greater bias effects for recognized items (.53) than for items they had failed to recognize (.37). The two proportions for the dichotic-listening condition were .38 for correct and .44 for incorrect recognition.

These results were generally consistent with the hypothesis that attentive processing during encoding is more likely to invite analytic, conceptually-driven processing during retrieval. The absence of stochastic independence for the question condition confirms that the subjects relied on similar analytic processes for both implicit and explicit tasks. The question condition provided a better basis for analytic processing during recognition because the original encoding context (questions) could be more easily retrieved, as opposed to the limited context provided by critical phrases (only) in dichotic listening. Furthermore, an implicit task may be most likely to invite analytic processing when attention has been allocated to the first exposure and when the second exposure (recognition) has evoked further analytic processing. Schacter's (1987) recent review also suggests that the elaborative processing demanded by our question condition may have led to a kind of "involuntary explicit memory" involving an "unintentional but fully conscious and explicit 'reminding' of the occurrence of a prior episode" (p. 510).

Further correlational analyses suggested some incompatibility between overall performances on explicit and 
implicit tasks for the subjects in the dichotic-listening condition. That is, a significant negative correlation between total recognition and total implicit scores (number of biased interpretations for old homophones) was obtained for the subjects in the dichotic-listening condition $[r(46)=$ -.32 ], but not for the subjects in the question condition $(r=.10)$. The subjects who showed the strongest biasing effects in implicit testing were those who had performed relatively poorly in the preceding recognition test. The most plausible explanation for this negative correlation appears to be that the subjects in the dichotic-listening condition relied on nonanalytic processes for both tasks. Clearly, implicit scores reflected the extent to which the subjects were effectively biased toward the least-frequent meanings of old homophones. Such effective biasing could sometimes interfere with effective recognition if subjects rely on a nonanalytic fluency heuristic. That is, effective biasing could lead subjects to reject some correct homophone choices (during recognition) simply because their biased (least-frequent) meanings seem relatively unfamiliar. Of course, the feeling of familiarity arising from the frequency of prior processing (prior to the experimental exposure; see Jacoby, 1988) would be greater if homophones were interpreted in terms of their most-frequent, rather than least-frequent (biased), meanings. If something seems unfamiliar because it has seemed unfamiliar in the past, we may erroneously conclude that it must not have been in the prior episode (e.g., experimental exposure). As Jacoby (1988) notes, the fluency heuristic is vulnerable to error precisely because extraneous factors enhance perceptual fluency (e.g., word frequency).

If the subjects were relying on nonanalytic processing for both retrieval tasks (implicit and explicit) following unattentive encoding, it seems that stochastic dependence should have been as apparent for the dichotic-listening condition as it was for the question condition. Although not reliable, the two implicit proportions (reported previously) for the dichotic-listening condition did indeed differ in the expected direction. That is, greater biases for the implicit task were apparent for items following incorrect $(44 \%)$, as opposed to correct ( $38 \%)$ recognition. The same implicit proportion scores were calculated for subject groups obtained through the application of increasingly stringent criteria for good recognition. As can be seen in Table 1, stochastic dependence (i.e., higher implicit proportions for items not recognized) became increasingly apparent as recognition got better. (The pattern was strikingly different for the question condition.) As suggested by these trends, the correlation between total recognition and proportion difference scores (reflecting stochastic dependence; see Table 1) was significant for the dichoticlistening condition $[r(46)=-.30]$, but not for the question condition $(r=.01)$. The subjects who generally evoked dominant meanings for homophones-thereby, doing well in recognition-appear to have been the most negatively affected (in implicit tasks) by their own recog-
Table 1

Mean Proportions of Correct Implicit Responses Following Correct and Incorrect Recognition for Groups Varying in Recognition Accuracy

\begin{tabular}{|c|c|c|c|c|}
\hline \multirow{2}{*}{$\begin{array}{l}\text { Minimum } \\
\text { Recognition Score } \\
\text { for Inclusion }\end{array}$} & \multirow[b]{2}{*}{$n$} & \multicolumn{2}{|c|}{ Implicit Proportions } & \multirow[b]{2}{*}{ Difference } \\
\hline & & $\begin{array}{c}\text { Correct } \\
\text { Recognition }\end{array}$ & $\begin{array}{c}\text { Incorrect } \\
\text { Recognition }\end{array}$ & \\
\hline \multicolumn{5}{|c|}{ Dichotic-Listening Condition } \\
\hline $\begin{array}{l}4 \\
5 \\
6 \\
7\end{array}$ & $\begin{array}{r}40 \\
26 \\
13 \\
4\end{array}$ & $\begin{array}{l}.34 \\
.37 \\
.33 \\
.28\end{array}$ & $\begin{array}{l}.45 \\
.48 \\
.58 \\
.75\end{array}$ & $\begin{array}{l}-.11 \\
-.11 \\
-.25 \\
-.47\end{array}$ \\
\hline \multicolumn{5}{|c|}{ Question Condition } \\
\hline $\begin{array}{l}4 \\
5 \\
6 \\
7\end{array}$ & $\begin{array}{l}44 \\
39 \\
25 \\
16\end{array}$ & $\begin{array}{l}.54 \\
.53 \\
.49 \\
.44\end{array}$ & $\begin{array}{l}.36 \\
.36 \\
.40 \\
.31\end{array}$ & $\begin{array}{l}.18 \\
.17 \\
.09 \\
.13\end{array}$ \\
\hline
\end{tabular}

nition successes; those successes might have reinforced the most-frequent, rather than biased, meanings of old homophones.

Overall, the results of this experiment suggest that attentive encoding evoked analytic processing and that unattentive encoding evoked nonanalytic processing in later retrieval tasks (whether implicit or explicit). When encoding conditions facilitate the processing of infrequent meanings and invite a fluency heuristic (for explicit tasks), it should not be surprising to find evidence for remembering only when subjects are unaware of remembering (i.e., given implicit tasks; Eich, 1984). Under these circumstances, effective semantic processing can have very different consequences for implicit and explicit tasks. For the present experiment, evidence for such differential consequences was not restricted to analyses of responses to the $\mathbf{8}$ homophones presented in both tasks (as previously reported). Even when implicit scores reflected responses to items not tested in recognition, a significant negative correlation between recognition and implicit scores was obtained for the subjects in the dichotic-listening condition $[r(46)=-.34]$.

Finally, there was a surprising degree of similarity for results obtained from the two implicit tasks (spelling and free association). This similarity is importnat in the context of suggestions that various implicit tasks may be tapping different aspects of implicit memory (Schacter, 1987). Our results indicate that the essential dimension to consider when characterizing an implicit task is the extent to which the overall experimental conditions invite nonanalytic processes, rather than specific aspects of the implicit task itself.

\section{REFERENCES}

EICH, E. (1984). Memory for unattended events: Remembering with and without awareness. Memory \& Cognition, 12, 105-111.

Galbraith, G. G., \& Taschman, C. S. (1969). Homophone units: A normative and methodological investigation of the strength of com- 
ponent elements. Journal of Verbal Learning \& Verbal Behavior, 8 , 737-744.

JACOBY, L. L. (1988). Memory observed and memory unobserved. In U. Neisser \& E. Winograd (Eds.), Real events remembered: Ecological approaches to the study of memory. Cambridge, England: Cambridge University Press.

JACOBY, L. L., \& BROOKS, L. R. (1984). Nonanalytic cognition: Memory, perception, and concept learning. The Psychology of Learning \& Motivation, 18, 1-47.

JACOBY, L. L., \& DALlaS, M. (1981). On the relationship between autobiographical memory and perceptual learning. Journal of Experimental Psychology: General, 110, 306-340.
JACOBY, L. L., \& WITHERSPOON, D. (1982). Remembering without awareness. Canadian Journal of Psychology, 36, 300-324.

SCHACTER, D. L. (1987). Implicit memory: History and current status. Journal of Experimental Psychology: Learning, Memory, \& Cognition, 13, 501-518

SCHACTER, D. L., \& GRAF, P. (1986). Effects of elaborative processing on implicit and explicit memory for new associations. Journal of Experimental Psychology: Learning, Memory, \& Cognition, 12, 432-444.

(Manuscript received June 4, 1988.) 